Published in Revue de médecine périnatale, 2019, vol. 11 no. 1, p. 4-8 which

should be cited to refer to this work

DOI : $10.3166 / \mathrm{rmp}-2019-0042$

Repenser les soins au post-partum : mise en perspective d'un véritable plan périnatal

Benzonana $\mathbf{P}^{1}$; Epiney $\mathbf{M}^{2}$; Razurel $\mathbf{C}^{3}$

1Pascale Benzonana

Sage-femme responsable de l'unité post-partum, Département de gynécologie et d'obstétrique

Hôpitaux universitaires de Genève

Département de Gynécologie et d'Obstétrique

Bd. de La Cluse 30

CH-1211 Genève 14

Pascale.Benzonana@hcuge.ch

${ }^{2}$ Manuella Epiney :

Médecin adjointe agrégée, responsable de l'unité de périnatalité, Département de gynécologie et d'obstétrique

Hôpitaux universitaires de Genève

Département de Gynécologie et d'Obstétrique

Bd. de La Cluse 30

CH-1211 Genève 14

Manuella.Epiney@hcuge.ch

${ }^{3}$ Chantal Razurel

Professeure associée, Sage-femme, $\mathrm{PhD}$ en psychologie, Haute Ecole de Santé de Genève

Haute Ecole de Santé

47 avenue de champel

CH-1206 Genève

Chantal.razurel@hesge.ch 


\section{Repenser les soins au post-partum : mise en perspective d'un véritable plan}

\section{périnatal}

\section{Revisiting postpartum care: putting in perspective a real perinatal plan}

\section{Résumé :}

La naissance entraine des bouleversements pouvant impacter la santé psychique de la mère et le processus d'attachement avec l'enfant. La prise en charge faite lors de la période postpartum peut grandement influencer ces issues.

Une approche centrée sur les besoins particuliers des mères et s'appuyant sur des entretiens psycho-éducatifs pour mobiliser leurs ressources, a été menée à la maternité des HUG (Genève). Cette action a montré une augmentation du sentiment de compétence des mères. Ceci vient s'intégrer dans un véritable plan périnatal basé sur l'emporwerment des femmes.

\section{Abstract :}

Birth produces changes that can affect the mother's psychic health and the attachment process with the child. Postpartum care can greatly influence these issues.

An approach focused on the special needs of mothers and based on psycho-educational interviews to mobilize their resources, was conducted at the maternity hospital of HUG (Geneva). This action has shown an increase in mothers' sense of competence.

This is integrated into a real perinatal plan based on the emporwerment of women.

\section{Mots clés :}

Dépression périnatale, Postpartum, Sentiment de compétence, entretien psycho-éducatif, formation soignants.

\section{Key words :}

Perinatal depression, Postpartum, Sense of competence, psycho-educational interview, training caregivers. 


\section{Introduction}

La naissance d'un enfant entraine des bouleversements importants chez les mères ce qui les engagent dans un processus d'adaptation [1] pouvant entrainer une perception de stress [2]. Or ce stress perçu est fortement lié au risque de dépression périnatale [2,3] risque majeur dans le processus de la maternalité [4].

La période du postpartum précoce constitue un moment essentiel qui s'accompagne d'un taux élevé de stress [5]. Lors de cette période, chaque mère va avoir des besoins spécifiques et multi-dimensionnels [6] et attend d'être guidée, entourée et accompagnée pour développer son sentiment de compétence [7]. La littérature relève également qu'une approche individualisée centrée sur les perceptions et les besoins particuliers des mères, permet de développer un sentiment de sécurité [8]. Pourtant, la littérature a montré que l'offre en soins au postpartum peut se trouver éloignée des désirs et besoins des mères $[9,10]$. Plus particulièrement les mères considèrent que les soins ne sont pas individualisés et que leurs émotions ne sont pas prises en considération [9]. Les mères relèvent également que les informations qu'elles reçoivent sont souvent trop nombreuses dans un court laps de temps $[6,10]$.

C'est également le constat qui a pu être fait à la maternité des hôpitaux universitaire de Genève (HUG).

\section{L'expérience menée à Genève}

La maternité des hôpitaux universitaire de Genève, de type III, est la plus grande maternité de Suisse avec plus de 4100 naissances par année. Elle réalise $65 \%$ des accouchements du canton de Genève (500 000 habitants). Les autres accouchements ont lieu en cliniques privées mais également par des sages-femmes en maison de naissance ou à domicile. 
Le suivi de la grossesse est essentiellement effectué en libéral par des gynécologues ou des sages-femmes pour les grossesses physiologiques ou à la maternité (grossesse à haut risque ou dans le cadre du «suivi global» par une équipe de sages-femmes). La maternité encourage l'allaitement maternel (label «hôpital ami des bébés » décerné dès l'an 2000) avec un taux d'allaitement de $95 \%$, dont $82 \%$ d'allaitement exclusif. La durée de séjour au postpartum diminue et $95 \%$ des mères rentrent à domicile avec un accompagnement par des sagesfemmes indépendantes pris en charge par l'assurance de base jusqu'à 56 jours postpartum.

Les priorités du service d'obstétrique sont l'amélioration des soins et la sécurité du patient. Les analyses des situations ont montré à quel point la pratique, les compétences techniques et la communication sont essentiels pour la gestion adéquate des situations obstétricales [11]. C'est pourquoi la maternité a développé, dès 2012, un programme de simulation "SimMAT" pour la formation continue des équipes médico-soignantes afin d'améliorer les compétences des obstétriciens, sages-femmes, pédiatres et anesthésistes par des entrainements réguliers suivi d'analyse de leur pratique en particulier pour les situations d'urgence. Mais au-delà des situations d'urgence la question du suivi et de l'accompagnement des mères en particulier dans la période du post-partum s'est posée? Comment optimiser les soins durant cette courte période hospitalière ?

Le post-partum de la maternité des hôpitaux universitaires de Genève a une capacité de 57 lits répartis sur 2 étages. Avec un taux d'occupation de 93,4\% et une durée moyenne de séjour de 3,8 jours, la maternité a totalisé 30685 journées d'hospitalisations pour l'année 2017 et offre de spacieuses chambres à 2 lits réunissant confort et sécurité. Pourtant, malgré des locaux neufs et confortables et des dossiers informatisés permettant un suivi personnalisé des patientes, force est de constater que les soins ne semblent pas toujours répondre aux réels besoins des mères. 
Un projet qualité a donc été envisagé afin d'améliorer l'adéquation entre les besoins des femmes et l'offre en soin dans le post-partum précoce en s'appuyant sur le plan stratégique institutionnel dont le but est d'humaniser les soins, et de se recentrer sur le patient avec un mot d'ordre : "plus de temps pour les patients".

\section{Etat des lieux}

Avant de formaliser un plan d'action, une analyse de la pratique professionnelle a été menée dans le service post-partum auprès des soignants et des médecins. Il est apparu que les sagesfemmes avaient souvent de la difficulté à définir leur rôle entre soins, éducation, information, et soutien émotionnel. Ces observations rejoignent par ailleurs les résultats de la littérature [12]. Les médecins relèvent également la complexité de leur rôle dans le post-partum et s'interroge sur la médicalisation de cette période. On relève d'ailleurs dans la littérature que les soignants ont de la difficulté à trouver du sens à leur travail dans cette période du postpartum précoce [13]. Or, des recherches ont montré un lien étroit entre la satisfaction du personnel en milieu hospitalier et la satisfaction des patients $[14,15]$.

\section{Les objectifs du projet}

Il est donc apparu que si l'objectif principal était d'améliorer la satisfaction des mères vis-àvis de leur prise en charge, un deuxième objectif était incontournable : le fait d'améliorer la satisfaction des soignants et médecins vis-à-vis de leur rôle professionnel. Il est apparu essentiel d'associer sages-femmes et gynécologues dans l'élaboration et la mise en place du projet.

\section{Le plan d'action}


On constate que si les études montrent la nécessité de mettre en place un suivi personnalisé centré sur les besoins particuliers des mères dans cette période du post-partum précoce pour améliorer leur adaptation, il manque des modèles de soins pour atteindre cet objectif. C'est pourquoi un entretien psycho-éducatif spécifique pour la période périnatale a été développé et a été utilisé comme modèle de prise en charge.

\subsection{Formation des professionnels}

\subsubsection{L'outil d'intervention}

L'entretien psycho-éducatif a pour but de se centrer sur les mères afin d'augmenter leur sentiment de compétence en leur permettant de trouver des solutions correspondant à leurs besoins et en potentialisant leurs ressources [16]. Cet entretien suit 4 étapes :

- La première étape a pour but de mettre en évidence le stress perçu par les femmes. Cette étape nécessite de faire émerger les événements auxquels la mère est confrontée (en évoquant les facteurs de stress mis en évidence dans l'échelle de stress PDPSI [5] et d'évaluer si ces événements dépassent ses ressources et provoquent du stress.

Si effectivement ces événements provoquent un stress l'étape 2 de l'entretien est conduite.

- La deuxième étape a pour but de changer la perception de l'événement en travaillant sur les représentations/croyances et en amenant des éléments d'éducation pour intercéder sur l'évaluation. Cette étape nécessite d'investiguer les représentations, en lien avec la perception des événements, de déconstruire ce lien et d'apporter des éléments de connaissance qui permettent de voir l'événement sous un autre angle, moins menaçant pour la mère.

- La troisième étape a pour but d'amener les mères à développer d'autres manières de faire face aux événements auxquels elles sont confrontées, plus optimisées, à la lumière des éléments discutés et d'utiliser des stratégies plus fonctionnelles 
Pour terminer, la littérature a montré que la perception du soutien social peut influencer les effets du stress en modérant son impact sur la santé psychique des mères [17]. Cette quatrième étape a donc pour but d'augmenter la satisfaction du soutien social en regard des attentes des mères en les amenant à exprimer leurs besoins vis-à-vis de leur entourage et des professionnels pour ajuster l'adéquation du soutien-social.

Cet entretien psycho-éducatif peut donc être mené tous les jours du séjour hospitalier postpartum auprès de chaque mère comme l'a préconisé la recherche de Schmied [18] .

\subsubsection{Le déroulement de la formation}

Une formation théorique de deux jours a été menée auprès des professionnels (sages-femmes et médecins) pour leur permettre d'intégrer les techniques de l'entretien psycho-éducatif.

Le premier jour a consisté à poser les concepts théoriques et de présenter la trame d'entretien. Chaque étape a été travaillée par un training sur la base de vignettes cliniques et de jeu de rôle. Le deuxième jour a pour but de s'approcher le plus possible de situation du terrain pour exercer les compétences à acquérir. Ce travail s'est fait sur la base de pratiques simulées. Chaque situation a été jouée individuellement par les participants et une séance de débriefing avec l'ensemble du groupe a permis de discuter, d'ajuster et d'intégrer les notions liées à l'entretien psycho-éducatif.

Ensuite une supervision pour chaque participant a été programmée par la formatrice sur le terrain du professionnel. Cette supervision a également été suivie par un débriefing.

Enfin, une analyse de la pratique a été menée avec le groupe pour permettre une intégration des différentes notions. Ces séances sont organisées autour d'une situation rencontrée par les soignants dans leur exercice et est analysée par le groupe pour permettre de comprendre les obstacles ou les facilitations rencontrées. 
La formatrice qui a mené cette formation est sage-femme, docteure en psychologie et est titulaire d'un master en sciences de l'éducation. Elle est également l'auteure de l'entretien psycho-éducatif périnatal [16].

\subsection{Organisation du travail}

Néanmoins, lors de l'analyse de situation, les soignants relèvent l'inquiétude de « rajouter » un acte (avec l'entretien psycho-éducatif) alors que les conditions ne le permettent pas. C'est pourquoi ce projet s'accompagne également d'une réflexion pour réorganiser le travail autour de l'entretien psycho-éducatif dans un but d'intégration plutôt que dans une logique de juxtaposition. Cette réorganisation passe par deux axes : une réflexion concernant les profils et les spécificités des femmes et un deuxième axe concernant la répartition du travail selon les besoins particuliers des femmes.

\subsubsection{Les itinéraires cliniques}

Le rôle de l'itinéraire clinique est de détailler les étapes de prise en charge d'une situation (actes, durée, objectif et acteurs impliqués dans le processus) pour permettre l'efficience des soins en les adaptant aux patients. L'organisation et les pratiques sont ainsi orientées vers les intérêts et besoins particuliers des patients. L'un des deux objectifs en est la maitrise des coûts. La durée du séjour est ainsi «standardisée » ce qui induit une maitrise du nombre de jour d'hospitalisation, qui tend vers la diminution. Le second objectif reste la qualité, avec un patient impliqué dans le processus comme partenaire de soins. Cette organisation prend en compte le contexte spécifique de l'hôpital tout en se basant sur les bonnes pratiques (guidelines for clinical practice NICE [19] ). 
Les itinéraires cliniques au post-partum ont ainsi permis d'identifier les soins pertinents et ceux qui étaient superflus en fonction des risques et des besoins des patients par exemple concernant la durée de séjour des patientes, les examens de sortie physiologiques pris en charge par les sages-femmes, les examens de sortie du pédiatre... tout cela en collaboration et coordination entre sages-femmes, obstétriciens et pédiatres. Les soins orientés vers les besoins des patientes sont devenus ainsi la priorité en prenant en compte aussi bien la santé somatique que psychique.

\subsubsection{La répartition du travail et le travail en pluridisciplinarité}

La complexité des soins au post-partum tient également au fait que les rôles des différents professionnels peuvent ne pas être clairement définis. Or les différents professionnels sont nombreux à gravité autour de la mère, l'enfant, le couple... Les sages-femmes, les assistants en soins et santé communautaires (ASSC) ou aides-soignants, les gynécologues-obstétriciens, les pédiatres et dans certains cas les assistantes sociales ou les infirmières en pédiatrie....Si cette complexité peut se retrouver dans d'autres unités de soin, la période du post-partum hospitalier a la particularité de ne pas être centrée sur des pathologies (même si bien sûr cela peut arriver dans certains cas) mais bien de s'axer sur le maintien de la physiologie ce qui pose un contexte particulier. Cela entraine alors un flou quant au positionnement de chacun et au rôle spécifique de chaque professionnel. L'entretien psycho-éducatif peut alors être un outil performant pour permettre une répartition du travail avec les actes délégués objectivés.

On peut donc dire que l'entretien psycho-éducatif sera le point de départ d'une évaluation particulière en permettant de mettre en évidence les besoins des femmes et leurs risques, clarifiant ainsi les itinéraires cliniques. Par ailleurs, cela peut permettre d'éviter un éclatement des actes en ayant une évaluation commune et en permettant de planifier des actions 
raisonnées et concertées, d'où une limitation de la perte d'information ou de la répétition de mêmes informations. Ceci permet également d'améliorer la continuité des soins et de diminuer le sentiment chez les mères d'un découpage et de contradictions dans les propos et dans les prises en charge. Comme le disait une soignante formée à cette approche : «c'est prendre du temps supplémentaire pour en gagner à d'autres niveaux ».

\section{Autres expériences}

Un projet similaire a été également mené dans une autre maternité suisse au centre hospitalier universitaire Vaudois (CHUV). Une évaluation menée après cette intervention a pu mettre en évidence que les soignants relèvent une amélioration dans l'organisation des soins dans cette période d'hospitalisation post-partum. On observe que l'utilisation de l'entretien par les soignants s'est faite de plus en plus systématique et est conduite tous les jours sur une durée de 15 à 20 minutes. Ce résultat est très important et montre l'adéquation de cet outil pour tous les soignants [12]. Néanmoins comme nous l'avons évoqué ci-dessus, la mise en place de cette approche s'est accompagnée d'une réorganisation du travail tant au niveau des itinéraires cliniques des mères que dans la clarification des rôles de chacun : le travail au postpartum s'étant organisé autour de binômes Soignants/ASSC pour permettre le développement d'une véritable philosophie de soin centrée sur les besoins des mères. Les résultats montrent également une revalorisation du travail dans le post-partum avec une baisse du sentiment de frustration chez les soignants [12]. Enfin les résultats montrent les effets positifs de l'intervention psycho-éducative sur l'adaptation des mères qui se sentent moins inquiètes et moins stressées de manière significative [12]. C'est également ce que montre la recherche de Schorey [20] qui met en évidence qu'une intervention psycho-éducative dans le post-partum augmente le sentiment d'auto-efficacité des mères et réduit le risque de dépression à 6 et 12 mois post-partum. Néanmoins on peut noter que les mères regrettent que cette approche ne 
soit pas utilisée par les médecins, ceux-ci n'ayant pas été formés lors du projet développé au CHUV (ce qui n'est pas le cas dans le projet développé aux HUG pour lequel les médecins sont intégrés).

\section{Vers un suivi global du continuum de la naissance}

L'analyse du travail met également en évidence un manque d'efficience dans les liens avec le réseau extrahospitalier avec l'impression d'une discontinuité dans la prise en charge.

Une autre action serait donc de compléter le dispositif de l'hospitalisation post-partum avec un entretien prénatal et un entretien postnatal à plus long terme. Cela pourrait contribuer par ailleurs à définir les itinéraires cliniques évoqués ci-dessus, en mettant en évidence de manière plus claire les besoins et les risques des patientes tôt dans la grossesse et dans le postpartum à plus long terme. Les études montrent l'intérêt de mettre en place un suivi pré et postnatal sous la forme d'un entretien [21]. Une recherche a par ailleurs montré qu'un entretien psycho-éducatif mené en pré et en postnatal permettait d'améliorer la santé psychique des mères, la qualité du lien mère-enfant ainsi que le sentiment de compétence des mères [22].

\section{Conclusion et perspectives}

C'est donc dans un véritable plan périnatal global que s'insère cette expérience aux HUG. C'est par ailleurs ce qui est en réflexion à Genève actuellement, soutenu par la direction générale de la santé de l'état de Genève, pour penser un véritable continuum de la naissance en intégrant un entretien pré et postnatal et une prise en charge centrée sur les mères afin d'optimiser la santé des mères, des couples et des familles [23]. Ce dernier point viendrait compléter la pratique développée dans la période du postpartum hospitalier et permettrait 
d'ancrer une véritable philosophie de soin centrée sur les besoins particuliers des mères et des familles en articulant les différents intervenants et institutions autour de la mère.

\section{Références}

[1] Razurel C, Bruchon-Schweitzer M, Dupanloup A et al (2011) Stressful events, social support, and coping strategies of primiparous women during the postpartum period: a qualitative study. Midwifery 27:237-242.

[2] Razurel C, Kaiser B, Sellenet C, Epiney M (2013) Relation between perceived stress, social support and coping strategies and maternal well-being: a review of the literature. Women \& Health 53:74-99.

[3] Razurel C (2012) Facteurs psychosociaux ayant un impact sur la santé psychique des mères. Journal Suisse de La Sage-Femme Mai:13-14.

[4] Nanzer N (2009) La dépression postnatale: sortir du silence. Lausanne: Favre.

[5] Razurel C, Kaiser B, Dupuis M et al (2014) Validation of the post-delivery perceived stress inventory. Psychology, Health \& Medicine;19:70-82.

[6] Rudman A, El-Khouri B, Waldenström U (2008) Evaluating multi-dimensional aspects of postnatal hospital care. Midwifery 24:425-41.

[7] Razurel C, Desmet H, Sellenet C (2011) Stress, soutien social et stratégies de coping: Quelle influence sur le sentiment de compétence parental des mères primipares? Recherche En Soins Infirmiers 106:47-58.

[8] Wiklund I, Wiklund J, Pettersson V, Boström AM (2018) New parents' experience of information and sense of security related to postnatal care: A systematic review. Sex Reprod Healthc;17:35-42. 
[9] Hildingsson I.M (2007) New parents' experiences of postnatal care in Sweden. Women and Birth 20:105-13.

[10] Fenwick J, Butt J, Dhaliwal S et al (2010) Western Australian women's perceptions of the style and quality of midwifery postnatal care in hospital and at home. Women and Birth 23:10-21.

[11] Jastrow M, Picchiottino P, Savoldelli G, Irion O (2013) Simulation en obstétrique. Rev Med Suisse 9:1938-42.

[12] Razurel C, Avignon V, Gerdy P, Bouton J (2016) Postpartum psycho-educational interviews to promote adaptation in new mothers: A preliminary study. Journal of Nursing Education and Practice 7. doi:10.5430/jnep.v7n2p1.

[13] Razurel C, Héliot C, Perier J, Andreoli N (2003) Education des mères à la santé dans le post partum à la maternité de Genève. Recherche En Soins Infirmiers 75:38-45.

[14] Duclay E, Hardouin JB, Sébille V et al (2015) Exploring the impact of staff absenteeism on patient satisfaction using routine databases in a university hospital. Journal of Nursing Management 23:833-41.

[15] McHugh M.D, Kutney-Lee A, Cimiotti J.P et al (2011) Nurses' widespread job dissatisfaction, burnout, and frustration with health benefits signal problems for patient care. Health Affairs 30:202-10.

[16] Razurel C (2015) Se révéler et se construire comme mère : l'entretien psycho-éducatif périnatal. Genève: Medecine et Hygiène.

[17] Razurel C, Kaiser B (2015) The Role of Satisfaction with Social Support on the Psychological Health of Primiparous Mothers in the Perinatal Period. Women \& Health 55:167-86. 
[18] Schmied V, Cooke M, Gutwein R et al (2009) An evaluation of strategies to improve the quality and content of hospital-based postnatal care in a metropolitan Australian hospital. Journal of Clinical Nursing 18:1850-1851.

[19] NICE (2006) Postnatal care: routine postnatal care for women and their babies. Clinical Guidelines. London: National Collaborating Centre for Primary Care.

[20] Shorey S, Chan SWC, Chong YS, He H-G (2015) A randomized controlled trial of the effectiveness of a postnatal psychoeducation programme on self-efficacy, social support and postnatal depression among primiparas. Journal of Advanced Nursing 71:1260-73.

[21] Gao L, Chan SW, Sun K (2012) Effects of an interpersonal-psychotherapy-oriented childbirth education programme for Chinese first-time childbearing women at 3-month follow up: Randomised controlled trial. International Journal of Nursing Studies 49:27481.

[22] Razurel C, Antonietti J-P, Rulfi F et al (2017) The impact of pre- and post-natal psycho-educational intervention on the construction of parenthood. Archives of Women's Mental Health 20:469-72.

[23] N. Nanzer,M. Epiney. (2015) L'entretien prenatal genevois: étude de faisabilité et de satisfaction pour les femmes enceintes. Rev Med Périnat. 